\title{
INTELIGENCIA ARTIFICIAL Y SU APLICACIÓN EN LA ADMINISTRACIÓN DE JUSTICIA ${ }^{1}$
}

\author{
Artificial intelligence and its application in the administration of justice
}

Wilson Yesid Suarez Manrique ${ }^{2}$

Georgina Isabel De León Vargas ${ }^{3}$

\section{RESUMEN}

En este artículo se pretende reflexionar acerca de las diferentes aporías que se presentan en la interrelación entre la inteligencia artificial y los procesos judiciales. De forma específica se cuestiona ¿cuáles son las estrategias de aplicación más eficientes de la inteligencia artificial, para la resolución de los procesos judiciales, dentro de administración de justicia, desde la perspectiva del derecho fundamental a la tutela judicial efectiva? Así las cosas, se busca determinar y ponderar las estrategias de aplicación de la inteligencia artificial, para la resolución de los procesos, tomando como marco referencial el derecho fundamental a la tutela judicial efectiva. Para lograr lo anterior, en primer lugar, se describirán los elementos esenciales de la relación entre inteligencia artificial y el derecho. Posteriormente, se analizarán los diversos canales de aplicación que ostenta la inteligencia artificial en el ámbito de administración de justicia.

Palabras Claves: inteligencia artificial, derecho constitucional, tecnología, congestión judicial, proceso.

\footnotetext{
${ }^{1}$ Articulo avance parcial de investigación del Proyecto aprobado en convocatoria interna, con código CURN 02-2019 de la Corporación Universitaria Rafael Núñez, campus Cartagena. Agradecimientos especiales a Diego Andrés Salgado Vivero, estudiante de III Semestre del Programa de Derecho de la Corporación Universitaria Rafael Núñez, integrante del Semillero de investigación SIFAD, del Programa de Derecho de la Corporación Universitaria Rafael Núñez, campus Cartagena.

${ }^{2}$ Doctor en Derecho Universidad de Salamanca, Docente Investigador del Programa de Derecho de la Corporación Universitaria Rafael Núñez, campus Cartagena, Integrante del Grupo de Investigación Derecho Público del Programa de Derecho de la Corporación Universitaria Rafael Núñez, campus Cartagena. investigador principal del Proyecto de investigación "Inteligencia Artificial y Derecho" aprobado por la Dirección de Investigación de la Corporación Universitaria Rafael Núñez bajo el código CURN 02-2019. https://orcid.org/0000-0002-1587-3926 E-mail: wilson.suarez@curnvirtual.edu.co

${ }^{3}$ Magíster en Derecho Laboral, Universidad Autónoma de Guerrero México, Docente con función de Coordinación de investigación del Programa de Derecho de la Corporación Universitaria Rafael Núñez, campus Cartagena, co-lider del grupo de investigación Derecho Público del Programa de Derecho de la Corporación Universitaria Rafael Núñez, campus Cartagena, co-investigadora del Proyecto "Inteligencia artificial y Derecho Constitucional" aprobado por la Dirección de Investigación de la Corporación Universitaria Rafael Núñez bajo el código CURN 02-2019, https://orcid.org/0000-0002-3277-5930 E-mail: georgina.deleon@curnvirtual.edu.co
} 


\begin{abstract}
In this paper we intend to study the different aporias emerging from the interrelation between artificial intelligence and judicial trials. Specifically, we investigate what the most efficient strategies of application of artificial intelligence might be, as for the resolution of judicial trials within judicial administration, from the perspective of the fundamental right to effective judicial protection. Thus, the aim is to determine and assess the strategies of application of artificial intelligence for the resolution of judicial trials, taking as a reference the fundamental right to effective judicial protection. To achieve the above, firstly, the essential elements of the relationship between artificial intelligence and law will be described. Subsequently, we will analyze the different ways of application of artificial intelligence in the field of judicial administration.
\end{abstract}

Keywords: Artificial intelligence, Constitutional Law, technology, judicial congestion, process.

\title{
INTRODUCCIÓN
}

Se trata, pues, de una tesis de dogmática constitucional en la cual los problemas son abordados desde la crítica racional de las estructuras normativas constitucionales, en donde se pretende su deconstrucción y reconstrucción con fundamento en la aplicación de la inteligencia artificial a los procesos judiciales, y la búsqueda de la interpretación constitucional más plausible, con referencia a la dignidad humana y a la protección de los derechos fundamentales. Los resultados de esta reflexión son trascendentales en la medida que inician a dilucidar caminos en Colombia para redefinir el derecho, su alcance, el poder de los ciudadanos, el empoderamiento tecnológico y la forma de participación democrática en los planes sociales. Desde un punto más concreto, permite buscar soluciones para la congestión judicial en la administración de justicia, permitir una protección más adecuada de los derechos fundamentales y presentar alternativas para el rediseño del aparato jurisdiccional.

La pregunta que condensa el problema de esta investigación es la siguiente: ¿cuáles son las estrategias de aplicación más eficientes de la inteligencia artificial, para la resolución de los procesos judiciales, dentro de administración de justicia, desde la perspectiva del derecho fundamental a la tutela judicial efectiva?

La tecnología es una herramienta fructífera y eficiente para la solución de distintos problemas en el derecho. La tecnología disponible, afecta los modos de pensamiento y expresión y crea un marco antropológico de cognición y comunicación dentro del cual se desenvuelve el derecho. (Susskind, 1996) Denominó que la "subestructura informacional" se compone de: (i) oralidad, (ii) escritura, (iii) imprenta, y (iv) tecnologías de la información. 
Edwina Rissland, en su célebre escrito “Artificial Intelligence and Law” definía la inteligencia artificial como "el estudio de los procesos cognitivos utilizando los marcos conceptuales y los instrumentos de la ciencia computacional" (Lillo Lobo, 2010). Las aplicaciones de estos procesos al derecho pueden sintetizarse, de manera general, en dos grandes bloques: "mejorar la gestión y el desempeño de las instituciones del sistema judicial en un sentido amplio" y "mejorar el vínculo existente entre el sistema judicial, y las diversas instituciones que lo componen y la ciudadanía, mejorando el nivel de acceso a la justicia."

Se ha debatido mucho sobre el juez artificial, la posibilidad de que sea un programa, y no una persona humana, quien dicte sentencia. Estas discusiones por lo general apuntan a direcciones equivocadas. Pues, este tipo de programas sobre el "juez autómata", no se refieren, especialmente, a que sea una maquina la que remplace la labor del juez, sino que se refiere a que existan programas de ayuden o que hagan más eficiente la toma de decisiones judiciales. Esta labor puede enfocarse desde diversas direcciones.

La aplicación de los programas autónomos o tecnológicos en áreas como la medicina y las finanzas ha sido importante, debido a la información que pueden manejar, los datos que pueden analizar y las facultades calculatorias que pueden tener. Los beneficios de esta conmutación pueden hacer más eficiente, entre otras cosas, la administración de justicia y la protección de derechos fundamentales.

El derecho es un objeto de aplicación tecnológica que puede tener frutos muy interesantes y problemas muy profundos. La aplicación de la tecnología ostenta la capacidad de trasformar los diversos subsistemas jurídicos. Algunos campos relacionados con el derecho financiero o con las telecomunicaciones han venido aplicando de manera pionera adelantos que interiorizan la relación entre derecho e inteligencia artificial.

Pese a lo expedito del trámite se estructuran problemas de falla en el razonamiento, de falta de fundamentación de las premisas, de decisiones incoherentes, contradictorias, que han perdido vigencia. La aplicación de la inteligencia artificial al campo del derecho constitucional, y para el caso, el de la resolución de los procesos judiciales, plantea retos importantes en torno a la tutela judicial efectiva, el juez natural, la celeridad, la legalidad, y la neutralidad judicial.

Así pues, el uso de las tecnologías de la información en la administración de justicia puede suponer importantes beneficios: los profesionales de la justicia pueden ahorrar tiempo y trabajo, se obtiene mayor información y transparencia sobre el funcionamiento de la justicia, de manera más eficaz y eficiente; las personas pueden relacionarse directamente con la justicia, lo que les puede facilitar el 
acceso a la misma.

De esta forma “... se puede señalar en términos generales que las TIC pueden posibilitar grandes ahorros de costos y de tiempos, mediante la automatización de lo repetitivo, el acceso más rápido y seguro a datos, la comunicación más fluida y segura, entre otros aspectos”. (Lillo Lobo, 2010)

Las actividades que responden a patrones recurrentes tareas repetitivas pueden automatizarse y permitir una estructuración de actividades más eficiente. Luego la gestión del conocimiento jurídico, la aplicación de tecnologías de la información, tiene una aplicación inmediata, puesto que permite una mejor descripción, clasificación, y gestión de los contenidos jurídicos en las grandes bases de datos, y un acceso más fácil a los datos jurídicos diseminados en la red. (Suarez Manrique, 2019)

\section{METODOLOGÍA}

Esta investigación, desde una perspectiva metodológica, en razón a su naturaleza, puede considerarse como un estudio jurídico, integrativo y abstracto de la relación entre inteligencia artificial y el constitucionalismo contemporáneo. Se trata de un estudio jurídico por que toma como objeto central de estudio las normas jurídicas -en sentido amplio- y su tensión con la inteligencia artificial. Es integrativo en la medida que comunica argumentos jurídicos con disciplinas exógenas siempre y cuando se traduzcan adecuadamente al lenguaje jurídico. Es una reflexión presentada en un grado de abstracción superior al de las teorías generales y específicas, cuyos alcances se pueden aplicar a la deliberación de la mayoría de los problemas de los derechos fundamentales que se presentan en los distintos constitucionalismos actuales.

Se trata, pues, de una tesis de dogmática constitucional en la cual los problemas son abordados desde la crítica racional de las estructuras normativas constitucionales, en donde se pretende su deconstrucción y reconstrucción con fundamento en la aplicación de la inteligencia artificial a los procesos judiciales y la búsqueda de la interpretación constitucional más plausible, con referencia a la dignidad humana y a la protección de los derechos fundamentales.

La tesis se desarrolla especialmente desde un enfoque deductivo y relacional en tres momentos, $\mathrm{y}$ en tres dimensiones argumentativas diferentes. Se trata, en el primer momento, de determinar el contexto teórico que referencia la investigación. En este nivel se acentúan especialmente argumentos descriptivos. En un segundo momento, en un grado de abstracción menor, se reconstruyen las hipótesis específicas de relación entre inteligencia artificial y los procesos, que se entienden, fundan, tensionan, interactúan y comunican, con el contexto del primer momento, en este nivel se presentan argumentos especialmente analíticos. En el tercer momento, con base en el contexto y la reconstrucción del punto nodal, se evalúan y prescriben soluciones jurídicas en situaciones 
particulares. En este nivel son importantes los argumentos en su dimensión crítica o prescriptiva.

\section{Conceptualización y ámbito de Derecho Artificial.}

Para (Casanovas, 2010) la relación entre derecho y tecnología es antigua. La tecnología disponible, afecta los modos de pensamiento y expresión y crea un marco antropológico de cognición y comunicación dentro del cual se desenvuelve el derecho.

Richard Susskind denominó que la "subestructura informacional” se compone de: (i) oralidad, (ii) escritura, (iii) imprenta, y (iv) tecnologías de la información.

El derecho se tensiona y moldea de conformidad con el campo cognitivo y comunicacional en el cual se desarrolle. Distintas situaciones tecnológicas, sociales, culturales y económicas imprimen la necesidad a la administración de justicia de incorporar tecnologías de información para prestar un servicio más eficiente. Por ejemplo, problemas de mora judicial, de gestión de información, acceso a la justicia, "facilitar y hacer más efectiva la tramitación de causas; y, en general, mejorar la organización del trabajo y productividad de los tribunales, así como optimizar la calidad de la información que es producida en audiencia, entre otras. (Lillo Lobo, 2010).

Según (Agustí Cerrillo, 2012) los diferentes usos de las TIC, en la Administración de Justicia, pueden calificarse en cuatro categorías: tratamiento de la información, gestión de los expedientes judiciales, relaciones entre los operadores jurídicos y toma de decisiones.

El artículo 95 de la Ley 270 de 1996, establece que [e]l Consejo Superior de la Judicatura debe propender por la incorporación de tecnología de avanzada al servicio de la administración de justicia. Esta acción se enfocará principalmente a mejorar la práctica de las pruebas, la formación, conservación y reproducción de los expedientes, la comunicación entre los despachos y a garantizar el funcionamiento razonable del sistema de información. En la sentencia C-037 de 1996, la Corte Constitucional declaró condicionalmente exequible este artículo, señalando que:

\footnotetext{
"esta disposición busca que la administración de justicia cuente con la infraestructura técnica y la logística informática necesaria para el recto cumplimiento de las atribuciones y responsabilidades que la Constitución le asigna. Naturalmente, el uso de los medios que se encuentran a disposición de juzgados, tribunales y corporaciones judiciales exige una utilización adecuada tanto de parte del funcionario como de los particulares que los requieran".
}

Posterior a esta providencia se expide la Ley 527 de 1999, por medio de la cual se define y reglamenta el acceso y uso de los mensajes de datos, del comercio electrónico y de las firmas digitales, se establecen las entidades de certificación y se dictan otras disposiciones. En sentencia C-831 de 2001, la Corte señaló que: 
"La ley 527 de 1999 no se restringe a las operaciones comerciales sino que hace referencia en forma genérica al acceso y uso de los mensajes de datos, lo que obliga a una comprensión sistemática de sus disposiciones con el conjunto de normas que se refieren a este tema dentro de nuestro ordenamiento jurídico y en particular con las disposiciones que como el artículo 95 de la Ley Estatutaria de administración de Justicia se han ocupado de esta materia”.

En virtud de estas disposiciones jurídicas y de necesidades de índole constitucional, como el acceso a la tutela judicial efectiva, los poderes públicos ostentan el mandato de incorporar las tecnologías de la información y la comunicación a su quehacer, desde diferentes puntos y con diversas finalidades relacionadas entre sí.

Un tema de especial relevancia es el de la incorporación de la inteligencia artificial en el derecho constitucional, como un elemento de soporte para la toma de decisiones. En 1990, (Rissland, 1999), en su célebre escrito “Artificial Intelligence and Law” definía la inteligencia artificial como "el estudio de los procesos cognitivos utilizando los marcos conceptuales y los instrumentos de la ciencia computacional.

De acuerdo a Nieva $(2018$, p. 20) "no existe un total consenso sobre lo que significa la expresión de "Inteligencia Artificial", pero sí que podría decirse que describe la posibilidad de que las maquinas, en alguna medida, "piensen", o más bien imiten el pensamiento humano a base de aprender y utilizar las generalizaciones que las personas usamos para tomar nuestras decisiones habituales".

La relación entre la inteligencia artificial y el derecho es relativamente antigua y ambigua. Ostenta como finalidad solucionar un conjunto de problemas específicos que se plantean en el ámbito jurídico. Estos problemas refieren, al uso de los datos, la proyección de casos, el manejo y administración de los expedientes y la gestión de la información. Pamela Gray ha ordenado en años y etapas los sucesivos logros de estas aplicaciones que siguen en paralelo los avances de las corrientes principales de IA. Los temas se replican unos a otros, incorporándose a su vez al desarrollo del tema siguiente: (1) lenguaje jurídico, (2) lógica deóntica, (3) procesamiento por reglas, (4) procesamiento por casos, (5) estratificación del razonamiento, (6) razonamiento procedimental, (7) coordinación de tareas múltiples (Agustí Cerrillo, 2012).

Para Lobos las aplicaciones pueden sintetizarse, de manera general, en dos grandes objetivos. En primer lugar: "mejorar la gestión y el desempeño de las instituciones del sistema judicial (especialmente del Poder Judicial) en un sentido amplio". En segundo lugar: "la implementación de TIC puede tener por objeto generar o mejorar el vínculo existente entre el sistema judicial, y las diversas instituciones que lo componen y la ciudadanía, mejorando el nivel de acceso a la justicia. 
Algunos ejemplos paradigmáticos como Finlandia, Holanda, Portugal, Singapur, Israel e Inglaterra tienen sistemas jurídicos en los que las aplicaciones de tecnologías de la información han tenido una importante utilidad y han funcionado de manera adecuada.

El sistema Tuomas de Finlandia "contiene de manera virtual toda la información importante respecto a cada acción o causa ingresada al tribunal". Holanda ha sido un líder en el uso interno de tecnologías para brindar apoyo a la administración del tribunal y a jueces, y para el intercambio de información entre los tribunales y el público general. En Portugal “H@bilus” permite que el flujo del procedimiento judicial esté cubierto por aplicaciones informáticas; todas las notificaciones se hacen de forma electrónica y los operadores cuentan con una zona dedicada a las notificaciones recibidas en sus procesos con alertas automáticas. En Israel el NGCS es una red en la que se cargan los documentos y comunicaciones judiciales mediante un sistema de ingreso electrónico con el objeto de mejorar la eficiencia del sistema judicial. En Inglaterra el sistema XHIBIT permite la coordinación entre los diversos actores que lo componen, haciéndolo más rápido en el flujo de información esencial producida en la audiencia.

Respecto a la tramitación de causas, en Singapur existe el denominado EFS, para casos de menor relevancia, como aquellos casos no contenciosos, y el sistema denominado "Internet Videophone Services", servicio de video teléfono. En Inglaterra el sistema “County Court Bulk Center" donde se tramitan las causas de ejecución de deudas monetarias, de manera automática mediante una aplicación computacional. El sistema de Demanda Monetaria en Línea o MCOL permite a los consumidores y comerciantes, interponer sus demandas de cobro de dinero bajo un proceso completamente basado en tecnología Web. "Con la aplicación MCOL los demandantes pueden presentar la demanda, chequear el estado del proceso, y cuando corresponde, solicitar la sentencia y su ejecución". Finlandia permite el sistema “Tuomas", el cual ayuda los jueces a proyectar sus decisiones. Les permite acceder a la información contenida en los documentos ingresados, y la base de datos Tuomas e incluye modelos estándares de decisiones los cuales son pre programados en un sistema computarizado. (Vargas, 2011)

Desde una perspectiva especialmente teórica, las líneas generales de investigación de inteligencia artificial y derecho pueden categorizarse en cinco: (1) los sistemas multiagentes; (2) las instituciones electrónicas; (3) los sistemas computacionales dialógicos; (4) las investigaciones vinculadas a la Web Semántica y (5) al Linked Open Data. (CEPAL, 2016)

Un "MAS es un sistema compuesto por diversos agentes que interaccionan entre si y se dirigen a finalidades y problemas colectivos”. Según (Casanovas, 2010) desde 2005 aproximadamente, el impulso dado a los sistemas multiagente desde la IA ha propiciado una reactivación del interés por 
los sistemas normativos, por la estructura lógica de las normas, y por el comportamiento dinámico que muestran los agentes inteligentes en relación a ellas (Axelrod, 1997).

En el actual desarrollo de la argumentación es visible el influjo de la denominada "lógica informal" en los años ochenta y noventa, y del trabajo intenso de Douglas Walton en la recuperación de los sistemas dialécticos, basados en la calidad de los argumentos contradictorios enunciados en un proceso dialógico (entre dos interlocutores). Argumentación y computación son en la actualidad dos campos mutuamente convergentes, con el común denominador de la formalización y su aplicabilidad en sistemas de IA. Sea como sea, los distintos tipos de diálogo, el modelado de controversias, los sistemas dialécticos de argumentación, constituyen hoy una de las líneas sólidas de investigación, que resume, además, el trabajo hecho en AI \& D sobre generación de argumentos en los programas ya construidos (Sartor, 2006).

En cuanto a la WEB semántica el problema lo describe McCarthy nadie sabe cómo construir una base de datos general del sentido común que pueda ser usada por cualquier programa que necesite el conocimiento. Se requiere el conocimiento lo más preciso posible de un ámbito y se requiere escalar al conjunto material de habilidades conocidas por "sentido común" o conocimiento material para que las consultas a un sistema experto tengan sentido y se realice la correspondencia [matching] entre problemas y soluciones (Cfr. Kauffmann Arthur, 2007). La metodología de construcción jurídica conceptual en la WS se lleva a cabo mediante las denominadas ontologías jurídicas, es decir, las estructuras editadas que delimitan el alcance de los conceptos, relaciones e instancias de un determinado campo del derecho, y permiten que los programas lo extiendan y apliquen a partir de inferencias consideradas válidas o razonables.

En lo concerniente a la LOD, Vinculación de Datos en Abierto, con las redes sociales, la explosión no ha sido solamente demográfica, sino que ha venido de la acumulación exponencial de datos vertidos en la red y de la capacidad de buscarlos, reorganizarlos y reutilizarlos. La relación entre sujetos, datos y metadatos puede activarse para producir una gran base de datos relacional de conocimiento, al servicio de la gente. Un creciente número de proveedores de datos en los últimos tres años, que ha llevado a la creación de un espacio global de datos que contiene miles de millones de aserciones: la Web de Datos. No existe, pues, por si hubiera alguna duda, la división entre un mundo digital y el mundo real de los usuarios. La clave está en la clasificación, taxonomía, organización y reutilización del conocimiento desigualmente distribuido en la red, pero con un potencial enorme de ser focalizado en situaciones locales (Kennedy, 1999).

\section{Factores adicionales en la toma de decisiones judiciales.}

A pesar de que la toma de decisiones judiciales tenga en apariencia mucho que ver con la lógica, esto más que una realidad es un juego del lenguaje propio del ámbito jurídico dado que lo ajustada 
que se encuentra una solución al derecho no depende necesariamente del vínculo que tengan las palabras sino muchas veces los elementos ajenos a la lógica como es la autoridad, el contexto social, la situación política, las bases religiosas y la ética del juzgador y muchos otros elementos que podrían permear la existencia de fallos que pueden ser claramente ilógicos pero jurídicamente aplicables.

Ahora bien otro inconveniente que ha de tener en cuenta es que el testigo no es un observador científico, sino un simple espectador fortuito que no tiene los conocimientos necesarios para analizar y examinar todo lo percibido, lo que produce que la información sea como mínimo incompleta y adicionalmente a ello expuesta a sus emociones, creencias limites intelectuales y a la capacidad de sus sentidos.

Finalmente, no se sabe ni se sabrá lo que a ciencia cierta acontece en cada caso, ya que conocer la verdad no es la naturaleza de la administración de justicia, simplemente creerla, esto refleja la imposibilidad del conocimiento de la verdad real y a cambio solo la formación de una aceptada verdad procesal. En tal sentido, Luna (2019) plantea lo siguiente:

Con el estudio de las nociones analizadas ha quedado claro que la búsqueda de la verdad en el proceso judicial es un objetivo difícil de satisfacer en modo pleno, por lo cual se plantea que frente al proceso lo que se tiene es una verdad con carácter de validez. Así, para el proceso los hechos son conforme se han probado y no como ocurrieron en la realidad. (p. 56-57)

\section{CONCLUSIONES}

Las áreas en las cuales la Inteligencia artificial ha generado procesos disruptivos dentro del derecho son: procedimientos judiciales, pruebas y toma de decisiones, en el campo de los procedimientos judiciales según Jordi Nieva se usa la inteligencia artificial para automatizar etapas para la producción de la sentencia, como la búsqueda jurisprudencial de precedentes protocolos para la expedición de providencias judiciales y, de forma general los diferentes tramites automatizables que se llevan a cabo desde un proceso judicial. En el ámbito probatorio alude a los programas o algoritmos utilizados en el análisis de los hechos dentro de los procesos, la verificación de los testimonios, autopsias virtuales. En cuanto a la toma decisiones el papel de la inteligencia artificial resulta bastante interesante y complejo por cuanto el razonamiento clásico y complejo que realiza un juez es difícil de emular por un alegorismo luego de forma general la principal tarea de la inteligencia artificial se enfoca a la presentación de diferentes alternativas entre las cuales elige el juez.

Una de las principales ventajas de la inteligencia artificial en el derecho está enfocada en la celeridad para la resolución de los procesos judiciales. Lo cual implica descongestión judicial, tutela judicial efectiva y convivencia pacífica. 
Una de las consecuencias de la inteligencia artificial en el rol del jurista es la exigencia de profesionales más capacitados y condición alterna para la solución de los problemas, debido a que los roles más mecánicos y cotidianos pueden automatizarse con mayor facilidad.

Dentro de la aplicación de la inteligencia artificial al derecho resulta difícil la neutralidad axiológica o la liberación moral en la toma de decisiones, por cuanto si bien es cierto los algoritmos no están inmersos en estas limitaciones conceptuales, si están inmersas las personas que configuran los algoritmos.

El principal aporte de la inteligencia artificial en la toma de decisiones judiciales está en la presentación de las alternativas por cuanto su papel no es el de reemplazar al juez sino de auxiliarlo $\mathrm{y}$ fortalecerlo.

La inteligencia artificial en el derecho, pese a que ha incursionado desde la década de los ochenta, está en un periodo de desarrollo en el cual los avances se deslumbran con consecuencias disruptivas a mediano plazo, teniendo en cuenta los costos de su implementación, los cambios estructurales a nivel jurídico y las limitaciones de esta disciplina en el campo social del derecho.

Los diferentes programas que se han creado para la búsqueda de información, el establecimiento de premisas normativas, la interpretación de los hechos y la solución de los casos han acentuado las desigualdades existentes con las personas en situaciones económicas favorables.

La formación de las competencias a nivel universitario de los abogados tiene que referenciarse en la implementación de la inteligencia a artificial debido a que este es un factor disruptivo de la forma en que se ejerce el derecho. Que estos cambios no solamente están referidos a la enseñanza en pregrado sino a la incursión de los programas de posgrados.

La relación entre inteligencia artificial y derecho, si bien no es novedosa, es un campo interesante para adelantar planes de investigación interinstitucional que involucren la relación entre universidad estado y sociedad.

Se podría estar hablando de justicia del futuro y se justifica abordando lo siguiente, una administración de justicia vanguardista tiene que estar al paso con los últimos adelantos científicos y tecnológicos, en este sentido se debe entender que el derecho debe modernizarse constantemente, valiéndose de la tecnología y de los avances científicos para cumplir sus objetivos, herramientas como la internet, los expedientes digitales, el litigio en línea, las audiencias virtuales, las bases de datos, los sistemas expertos jurídicos, son indispensables para desarrollar una labor acorde al mundo actual y por supuesto alejada de errores, dado que la ciencia jurídica debe estar abierta a escoger cualquier avance tecnológico y científico que pueda representar alguna utilidad en el 
desempeño de la profesión.

Se espera que la inteligencia artificial en la administración de justicia ocasione que la demanda de justicia pueda ser cumplida en mejor medida y en menos tiempo que en la actualidad, reduciendo por supuesto conflictos sociales que en muchas ocasiones se producen por el mal funcionamiento del aparato judicial del país. Pero debemos entender también que por muy inteligentes y expertos que sean los sistemas jurídicos vanguardistas, no se puede pensar en un reemplazo de la figura humana del juez en sus labores de dictar sentencias o providencias judiciales, no obstante son herramientas altamente útiles que evolucionan constantemente ante los avances de la informática, la inteligencia artificial y otras ciencias, ayudando a los jueces, a los trabajadores del sistema, y en general a la defensa a realizar su labor de forma más sencilla, y no por ello restando calidad en el trabajo realizado, ya que estos programas tienen la capacidad de contener en sus bases de conocimientos, una cantidad de información que un ser humano no podría tener presente en todo momento, sumado a que la pueden relacionar o interpretar como lo haría un experto en la búsqueda de soluciones a problemas jurídicos.

En Colombia uno de los muchos temas que demandaría la utilización de un sistema experto jurídico en el proceso de alimentos, para la fijación, aumento, disminución o exoneración de la misma, esto se evidencia en la cantidad de procesos que llegan al poder judicial cada año, y ante una realidad social tan marcara del país como es la irresponsabilidad de los padres en otorgarle a los hijos la renta alimentaria que por ley les corresponde, los procesos por alimentos involucran las demandas de cualquier ser humano en búsqueda por supuesto de obtener la satisfacción de una necesidad de llevar una vida digna, en efecto los problemas de congestión judicial podrían verse mejorados con el uso de las nuevas tecnologías al servicio de los procesos entre ellas de un sistema inteligente que colaboraría con el juez y los demás trabajadores del despacho en la proyección de las alternativas y soluciones en frente de los distintos conflictos jurídicos.

Para finalizar se puede resumir que el uso de la inteligencia artificial en el Derecho Colombiano puede ser indispensable para el desarrollo del derecho en sus múltiples dimensiones, dado que con estas herramientas de tipo tecnológico, principalmente los sistemas expertos jurídicos, se logra que la administración de justicia realice sus funciones de forma más ágil, restando tiempo y sumando calidad de labor desempeñada.

\section{REFERENCIAS BIBLIOGRÁFICAS.}

Agustí Cerrillo. (2012). 'E-justicia: las tecnologías de la información y el conocimiento al servicio de la justicia iberoamericana en el siglo XXI'. Derecho y Política, 2012.

Axelrod, R. (1997). The complexity of cooperation: agent-based models of competition and 
collaboration. Princeton University, New Jersey,: Princeton studies in complexity.

Casanovas, P. (2010). Tecnología, Inteligencia Artificial y Web Semántica. Un mundo para todos y para cada uno. Manual de Filosofía y Teoría del Derecho Tomo I, Cap.XXV, Universidad Nacional Autónoma de México, México. México: En Jorge Fabra y Ezequiel Spector (Ed.),.

Castells, M. (15 de Junio de 2001). Internet y la Sociedad Red. Lección inaugural del programa de doctorado sobre la sociedad de la información y el conocimiento, 1-13.

Castells, M. (2014). El impacto de internet en la sociedad: una perspectiva global. En bbvaopendmind, 19 ensayos fundamentales sobre cómo internet esta cambiando nuestras vidas (pág. 471). Barcelona: bbvaopendmind. Obtenido de https:/www.bbvaopenmind.com/articulo/ el-impacto-de-internet-en-la-sociedad-una-perspectiva-global/

CEPAL. (2016). La nueva revolución digital. Santiago: Naciones Unidas. Obtenido de http://www.cepal.org/ es/publicaciones/38604-la-nueva-revolucion-digital-la-internet-consumo-la-internet-la-produccion

Kauffmann Arthur. (2007). Hermenéutica y derecho, en sentido actual de la filosofía el derecho. Granada: Editorial Comares.

Córdoba, P. J. (2009). Hacia la apropiación del gobierno electrónico: una mirada crítica sistémica. Revista Chilena de Administración Pública(14), 61-82. Obtenido de Dialnet-HaciaLaApropiacionDelGobiernoElectronico-3359032\%20(1).pdf

Electrónico, R. d. (2014). Plan de Gobierno Electrónico 2014-2017. Versión 1.0. Quito: Secretaría Nacional de la Administración Pública. Obtenido de https://ec.okfn.org/files/2014/12/ PlanGobiernoElectronicoV1.pdf

Kennedy, D. (1999). Libertad y restricción Judicial. Bogotá: Uniandes Klug, Ulrich. Lógica jurídica. Bogotá: Temis, 1998.

Lillo Lobo, R. (2010). El Uso de Nuevas Tecnologías en el Sistema Judicial: experiencias y precauciones. Brasilia: Centro de Estudios de Justicia de las Américas.

Luna Salas, F. (2019). Hechos, verdad y prueba. Compendio de Derecho Probatorio Contemporáneo. Editorial Ibañez. Colombia. 
Nieva Fenoll, J. (2018). Inteligencia Artificial y proceso judicial. Editorial Marcial Pons.

Rissland, E. (1999). “Artificial Intelligence and Law: Stepping Stones to a Model of Legal Reasoning”. Yale: Yale Law Journal, vol. 99.

Sartor, G. (2006). "Fundamental legal concepts: a formal and teleological characterisation”, Artificial Intelligence and Law. London.

Suarez Manrique, W. \&. (Marzo de 2019). El mito de la estabilidad constitucional. Vía Iuris, (26), 26, 1-32. Obtenido de Suarez Manrique, W., \& de León Vargas, G. (2019). El mito de la estabilidad constitucion https://revistas.libertadores.edu.co/index.php/ViaIuris/article/view/862

Susskind, R. ,. (1996). The Future of Law. Oxford: Oxford University Press.

UIT. (2016). www.itu.int. Obtenido de http://www.itu.int/es/mediacentre/Pages/2016-PR30.aspx

Vargas, D. C. (2011). El Gobierno Electrónico o e-Gobierno. Uni-Pluri/Versidad, 11(1), 1-16. Obtenido de http://www2.congreso.gob.pe/sicr/cendocbib/con4_uibd.nsf/ BD9EB0AFF79442F705257C170009C981/\$FILE/9711.pdf 